\title{
Viabilidad y germinación de semillas de Hechtia perotensis (Bromeliaceae)
}

\author{
Violeta Elizalde ${ }^{1}$, José Rodolfo García ${ }^{1}$, Cecilia Beatriz Peña-Valdivia ${ }^{1 *}$, Ma. Carmen Ybarra ${ }^{2}$, \\ Otto Raúl Leyva ${ }^{3} \&$ Carlos Trejo ${ }^{1}$ \\ 1. Postgrado en Botánica, Colegio de Postgraduados, Campus Montecillo, Carretera México-Texcoco, km 35.5, Estado \\ de México, 56230, México; violeta.elizalde@colpos.mx, garcianr@colpos.mx, cecilia@colpos.mx, \\ cecibetipv@gmail.mx, catre@colpos.mx \\ 2. Departamento de Ingeniería Agroindustrial, Universidad Autónoma Chapingo, Carretera México-Texcoco km 38.5, \\ Estado deMéxico, 56230, México; agroalim@correo.chapingo.mx \\ 3. Facultad de Ciencias Biológicas y Agropecuarias, Universidad Veracruzana. Camino a Peñuela s/n. C.P. Peñuela, \\ Amatlán de los Reyes, Ver., México; oleyva@uv.mx \\ * Correspondencia
}

Recibido 05-IV-2016. Corregido 26-VII-2016. Aceptado 26-VIII-2016.

\begin{abstract}
Viability and germination of Hechtia perotensis (Bromeliaceae) seed. Endemic populations of Hechtia perotensis have been described in Puebla and Veracruz, Mexico. Good quality seed collections can be used in conservation, research and ecological restoration. To evaluate seed quality of wild and endemic species, some compounds are used as effective promoters of germination, such as potassium nitrate $\left(\mathrm{KNO}^{3}\right)$ and gibberellic acid $\left(\mathrm{AG}^{3}\right)$, because they increase seed germination capacity and reduce latency. The triphenyl tetrazolium chloride (tetrazolium) test correlates seed viability because it is based on the activity of dehydrogenases in live tissues that catalyze mitochondrial respiration. The objective of this study was to obtain information on size and weight of capsules and seeds and seed germination and viability of $H$. perotensis, collected in Veracruz in the year 2012 and 2015. The hypotheses were 1) that seed germination and viability are independent of the year of collection, 2) that there is a tetrazolium concentration that can identify seed viability better than others, and 3 ) that pretreatment with $\mathrm{KNO}^{3}$ or $\mathrm{AG}^{3}$ improves seed germination. Seed germination was assessed using a completely randomized design with three treatments (control and the germination promoters $0.2 \% \mathrm{KNO}^{3}$ and 500 $\mathrm{mg} / \mathrm{L} \mathrm{AG}^{3}$ ), four treatments for the viability test (control, $0.2,0.5$ and $1.0 \%$ of tetrazolium) and six replicates for each treatment. A total of one hundred seeds for germination experiments, and 25 seeds for the viability test were used. The results between and within years were analyzed with ANOVA and multiple comparison with the Tukey test. The proportion of non-germinated seeds was quantified along with the number of normal and abnormal seedlings, seeds with viable embryo, seeds without embryo, and seeds with low or no viability. On average, for the 2012 collected sample, $36 \%$ had viable embryos, $7 \%$ had low viability, $24 \%$ were not viable and $33 \%$ had no embryo. This result was significantly different from the 2015 sample, for which $87 \%$ of seed showed viable embryos, $10 \%$ had low viability, $0 \%$ was not viable and $3 \%$ had no embryo. Seed germination was also significantly different between years (22 and $92 \%$ ) Pregerminative treatments did not improve germination. Seed germination and viability of $H$. perotensis significantly varied between years of seed collection. Rev. Biol. Trop. 65 (1): 153-165. Epub 2017 March 01.
\end{abstract}

Key words: Bromeliaceae, dormancy, Hechtia perotensis, seed, seed vigour, tetrazolium test.

México se encuentra entre las regiones biogeográficas neotropicales, y debido a su riqueza de especies y endemismos excepcionales (Sarukhán, Halffter, \& Koleff, 2009) es considerado un país megadiverso y ocupa el cuarto lugar en el mundo por la diversidad de plantas vasculares que posee (Llorente-Bousquets \& Ocegueda, 2008). Entre las especies de plantas que habitan México se reconocen cuatro categorías de distribución: las endémicas 
de México, las endémicas de Megaméxico I (especies que se distribuyen en México y el suroeste de EE.UU.), las endémicas de Megaméxico II (desde México hasta la depresión central de Nicaragua) y las de Megaméxico III (desde el suroeste de EE.UU., hasta la depresión central de Nicaragua) (Rzedowski, 1991).

La recolección de semillas de buena calidad permite su uso en conservación, investigación y restauración ecológica. La semilla es almacén de la diversidad genética de la población original. La germinación de las semillas de especies silvestres y endémicas es especialmente importante ya que esa transición sucede en las etapas tempranas de vida y establece el contexto para el desarrollo posterior; además, la extensión de los nichos de germinación, repercute en la amplitud ecológica y la distribución geográfica, así como en la selección natural de la especie (Baskin \& Baskin, 2008; El-Maarouf-Bouteau \& Bailly, 2008).

El género Hechtia Klotzsch (1835:401) es un miembro de la familia Bromeliaceae $\mathrm{y}$ es el único de la subfamilia Hechtioideae (Givnish, Millam, Berry, \& Sytsma, 2007). De acuerdo con Ramírez M., Jiménez, FernándezConcha, y Pinzón (2014) México alberga el mayor número de especies de este género, con aproximadamente $94 \%$ de ellas endémicas del país, que en su conjunto está restringido a Megamexico III, que el número de especies se estima en 65 , pero está variando rápidamente por el descubrimiento frecuente de especies nuevas y señalan que este género es uno de los integrantes más interesantes de las Bromelias mexicanas. Las poblaciones de Hechtia crecen principalmente en regiones áridas y semiáridas y en algunos casos forman matorrales rosetófilos extensos (Ramírez et al., 2014).

Específicamente, $H$. perotensis I. Ramírez \& Martínez-Correa se ha localizado en los estados de Puebla y Veracruz, México, donde crece en matorrales xerófilos en una franja altitudinal estrecha de 2400 a $2500 \mathrm{msnm}$. El epíteto específico hace referencia a una de las áreas en las cuales crece la especie, que es el Valle de Perote en Veracruz. Las plantas de H. perotensis se han descrito como hierbas arrosetadas, terrestres, de hasta $2 \mathrm{~m}$ de altura cuando tienen flor; las rosetas son globosas, compactas y densamente cespitosas, y pueden alcanzar $50 \mathrm{~cm}$ de altura y $40 \mathrm{~cm}$ de diámetro. En una colonia puede haber 50 o más rosetas acaules o con tallos inconspicuos (Espejo-Serna, López-Ferrari, Ramírez-Morillo, \& Martínez-Correa, 2007). Las semillas son de color pardo claro, fusiformes, ligeramente rugosas, oblongas, elipsoidales o fusiformes, circumaladas, con una ala extendiéndose en dos caudas terminales de alrededor de $1 \mathrm{~mm}$ de ancho y 3 $\mathrm{mm}$ de longitud.

La biología de las semillas de Hechtia se conoce poco (Espejo-Serna et al., 2007) y la información es escasa en la literatura especializada actual, probablemente debido a su importancia limitada para los humanos. Entre las excepciones, están las descripciones morfológicas de las semillas de $H$. nuusaviorum (Espejo-Serna et al., 2007) y H. confusa (Montes R., 2013); la evaluación de la germinación de las semillas de $H$. confusa y $H$. tehuacana en placas con agar, su fotoblastismo, reacción al estrés hídrico y al potencial osmótico del medio (Montes R., 2013); Escobedo (2012) utilizó semillas de $H$. schottii Baker en germinación para evidenciar la presencia de dos alas reducidas. Reyes (2015) señaló que las semillas del género Hechtia han sido útiles para la restauración ecológica por su resistencia a la sequía extrema y muchas de ellas tienen importancia ornamental; aunque el autor no especificó cuáles especies se recomiendan por su mejor capacidad germinativa. El conocimiento de esta última podría aportar información sobre la permanencia de las especies endémicas in situ, favorecer la conservación ex situ y los protocolos de producción de plantas (Toledo \& Wolf, 2008; Pereira, Andrade, Pereira, Forzza, \& Rodríguez, 2010). La germinación ex situ es una opción para la propagación de especies con valor comercial y para su preservación y conservación de las que forman parte de la riqueza florística de México, además de ser un instrumento para facilitar el estudio de sus procesos ecológicos y fisiológicos. 
Los promotores para la germinación son compuestos químicos que se utilizan para iniciar o incrementar la capacidad germinativa de semillas. El ácido giberélico $\left(\mathrm{AG}^{3}\right)$ aplicado exógenamente es efectivo en el control y activación de la germinación de semillas debido a su habilidad de interrumpir estados de latencia y reemplazar estímulos ambientales, como los de la luz, la temperatura o de ambas. Por esto, $\mathrm{AG}^{3}$ incrementó los porcentajes de germinación y disminuyó el tiempo para su inicio en semillas de diferentes especies, como Ferocactus acanthodes, F. wislizeni (Deno, 1994), Albizia spp. (Tigabu \& Oden, 2001), Capsicum annuum var. glabriusculum (Dunal) Heiser \& Pickersgill (García, Montes, Rangel, García, \& Mendoza, 2010) y Jaltomata procumbens (Cav.) J. L. Gentry (Saldívar-Iglesias, LagunaCerda, Gutiérrez-Rodríguez, \& DomínguezGalindo, 2010). El nitrato de potasio $\left(\mathrm{KNO}^{3}\right)$ se usa para disminuir la latencia y promover la germinación de las semillas (Siadat, Moosavi, Zadeh, Fotouhi, \& Zirezadeh, 2011). Zheng et al. (2009) señalaron que los compuestos con $\mathrm{N}$ pueden aumentar la germinación por ajuste de la tasa de intercambio $\mathrm{K}^{+} / \mathrm{Na}^{+}$e incremento de la actividad de la amilasa, producción de ATP, y respiración de las semillas.

Por su parte, la prueba de tetrazolio (cloruro de 2, 3, 5-trifenil-2H-tetrazolio) permite estimar la viabilidad de las semillas (International Seed Testing Association, 2010). El protocolo adecuado varía entre las especies y recolectas; no todas las semillas responden igual a la prueba, ya que se basa en la actividad de las deshidrogenasas de la respiración que reducen químicamente la solución incolora de tetrazolio a formazán (1, 3, 5-trifenilformazán), de color rojo-rosado (Glenner, 1990); de esta manera, es posible identificar tejido vivo (coloración roja), parcialmente dañado (tonalidades rosadas) o muerto (blanco) (International Seed Testing Association, 2010).

El objetivo de este estudio fue conocer el tamaño y biomasa de las cápsulas y semillas, y la germinación y viabilidad de las semillas de $H$. perotensis recolectadas en Veracruz, México, durante los años 2012 y 2015. La hipótesis fue que la germinación y la viabilidad de las semillas de $H$. perotensis son independientes del año de recolecta, que su germinación incre-

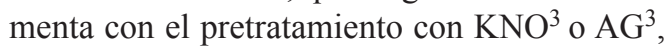
y que al menos una concentración de tetrazolio es adecuada para identificar su viabilidad.

\section{MATERIALES Y MÉTODOS}

Sitio de muestreo: Cápsulas de H. perotensis se recolectaron en septiembre 2012 y marzo 2015 de plantas de la región de Frijol Colorado, municipio de Perote, Veracruz, México (19³2'13.95" N, 97²2'98" O, 2416 msnm; geolocalización con Garmin eTrex 10 y Google Earth ${ }^{\circledR}$ ). El clima de la región de acuerdo con el sistema de clasificación climática de Köppen, modificado por García (1988), es semiárido, templado con veranos cálidos BS1kw(i')gw", con temperatura media mensual de $9.8^{\circ} \mathrm{C}$ en los meses más fríos, y $15.1{ }^{\circ} \mathrm{C}$ en los más cálidos; con una precipitación media mensual entre $11.2 \mathrm{~mm}$ en el mes más seco y $133.7 \mathrm{~mm}$ en el mes más húmedo. La vegetación del sitio de recolecta corresponde a un matorral xerófilo, también llamado izotal (Yuccapericulosa, Y. filifera y otras); el estrato arbóreo fue escaso y estuvo constituido por Nolina Michx., Yuca sp. y Dasylirion Zucc., en algunas zonas hubo árboles bajos, de menos de $5 \mathrm{~m}$ de altura, y esporádicos, pertenecientes a de los géneros Juniperus, Quercus y Pinus. En el sitio, las plantas de $H$. perotensis convivían con Agave horrida subsp. Perotensis B. Ulrich, D. acrotriche (Schiede) Zucc., Tillandsia erubescens Schltdl., T. recurvata (L.) L., N. parviflora (Kunth) Hemsl., Juniperus L. Q. microphylla Née, P. cembroides Zucc., Echeveria subalpina E. Walther, Salvia L., Lupinus L., Ephedra L., Sedum batesii L., Mammillaria magnimamma Haw., Coryphantha pallida (Engelm.) Lem., Cylindropuntia pallida (Engelm.) F.M. Knuth, Baccharis L., y algunas otras.

Peso y tamaño de las cápsulas y las semillas: Las cápsulas recolectadas se mantuvieron en refrigeración dos o tres semanas antes de su caracterización. La biomasa 
de 50 cápsulas se determinó en una balanza analítica ( $\pm 0.0001 \mathrm{~g}$; Scientech SA 120), su longitud y diámetro se midieron con un vernier digital ( $\pm 0.001 \mathrm{~mm}$; Mitutoyo). Las semillas de 150 cápsulas se contabilizaron, su biomasa se obtuvo en balanza analítica, y su longitud $\mathrm{y}$ anchura se midieron con vernier digital y microscopio estereoscópico.

Prueba de germinación con y sin nitrato de potasio y ácido giberélico como promotores de la germinación: Después de dos o tres semanas de las recolectas de cada año por separado, se realizaron las evaluaciones de germinación con seis repeticiones de cien semillas por tratamiento. Las semillas se desinfectaron externamente por inmersión en hipoclorito de sodio comercial (60 g/L de $\mathrm{Cl}$ activo) al $1 \%$ (v:v en agua), se enjugaron con agua destilada abundante y se depositaron en cajas Petri con papel filtro, humedecido con $20 \mathrm{~mL}$ de agua destilada (testigo), $\mathrm{KNO}^{3}$ (99\%; FERMONT $^{\circledR}$ ) al $0.2 \%$ (p:v en agua), $\mathrm{o} \mathrm{AG}^{3}(90 \%$; SIGMA $\left.^{\circledR}\right) 500 \mathrm{mg} / \mathrm{L}$. Las cajas se colocaron en una cámara con ambiente controlado (Thermo Scientific. EE.UU.; modelo 846) a $25 \pm 1{ }^{\circ} \mathrm{C}$ $\mathrm{y}$ fotoperiodo $12 \times 12 \mathrm{~h}(12 \mu \mathrm{mol} / \mathrm{m} / \mathrm{s}$ de irradiancia) (Sosa, Chávez, Mondragón, Estrada \& Ramírez, 2012). Las semillas se consideraron germinadas cuando el hipocótilo expuesto había alcanzado más de $1 \mathrm{~mm}$, se contabilizaron a los $8 \mathrm{~d}$ de establecido el ensayo y después de $15 \mathrm{~d}$ se obtuvo la germinación total (Montes R., 2013 observó que en ocho días, $H$. confusa alcanzó su germinación máxima) y el número de plántulas normales (sin deformaciones en las hojas) y anormales (plántulas con deformaciones en la primera hoja (International Seed Testing Association, 2010).

Prueba con tetrazolio: Las soluciones al $0.1,0.5$ y $1.0 \%$ de $2,3,5$ cloruro de trifenil tetrazolio (SIGMA-ALDRICH ${ }^{\circledR}$ ), las temperaturas y el tiempo de incubación de las semillas en la soluciones de tetrazolio se adaptaron según las recomendaciones para la prueba bioquímica de viabilidad por tetrazolio descritas en las reglas internacionales de análisis de semillas
(International Seed Testing Association, 2010). Cada tratamiento incluyó seis repeticiones y cada repetición se conformó de 25 semillas; así, el total de las semillas evaluadas en esta prueba fue 150 por tratamiento. Todas las semillas se preacondicionaron durante una noche $(16 \mathrm{~h})$ a $20 \pm 1{ }^{\circ} \mathrm{C}$ en rollos de papel absorbente (Scott Brand, Kimberly Clark) humedecido con agua. Luego, para propiciar la penetración de la solución de tetrazolio en los tejidos seminales, se realizó un corte horizontal arriba del embrión, con un bisturí con filo recto. Al realizar el corte, se cuidó de no dañar el embrión, y para esto se utilizó un microscopio estereoscópico (Leica EZ4 HD) (International Seed Testing Association, 2010).

Las semillas acondicionadas se colocaron en recipientes plásticos con tapa y se cubrieron con suficiente volumen de cada una de las soluciones de tetrazolio. Las muestras se mantuvieron en oscuridad, a $30 \pm 1{ }^{\circ} \mathrm{C}$, por $16 \mathrm{~h}$. Luego, las semillas se enjuagaron con agua destilada y los embriones se extrajeron raspando delicadamente la parte opuesta al corte horizontal que se realizó antes y empujando el embrión cuidadosamente con la punta de un bisturí; así, se obtuvieron los embriones completos. Los embriones se evaluaron y clasificaron en una de las clases de acuerdo con la "International Seed Testing Association” (2010).

Efecto de algunos factores ambientales en las características fisiológicas de las semillas: Con la finalidad de analizar el posible efecto de la temperatura, precipitación e irradiancia en las características fisiológicas de las semillas evaluadas, se obtuvo la información del clima de la región, entre 2011 y 2015, de la estación meteorológica Perote, del Laboratorio Nacional de Modelaje y Sensores Remotos del Campo Experimental Pabellón, del Instituto Nacional de Investigadores Forestales, Agrícolas y Pecuarias, México. Con los datos de precipitación y temperatura de los 12 meses previos a cada recolecta se elaboraron los climogramas de acuerdo con lo descrito por García (1988) y los datos de irradiancia se compararon entre los años con la prueba de $t$ de Student. 
Diseño experimental y análisis de resultados: Un diseño completamente al azar se utilizó para evaluar la germinación con tres tratamientos, un testigo, $\mathrm{KNO}^{3}(0.2 \%)$ y $\mathrm{AG}^{3}$ $(500 \mathrm{mg} / \mathrm{L})$. Seis repeticiones por tratamiento fueron incluidas con 100 semillas cada una; así, un total de 600 semillas fueron evaluadas en esta prueba. El análisis de normalidad se efectuó con la prueba Shapiro-Wilks; estas fueron analizados con ANDEVA, con la prueba de comparación múltiple de medias de Tukey ( $\alpha=$ $0.05)$ y con la prueba de $t$ de Student.

La prueba de tetrazolio se manejó con un diseño completamente al azar, con tres concentraciones, o tratamientos de tetrazolio $(0.2$, 0.5 y $1.0 \%$ ). Cada tratamiento incluyó seis repeticiones y en cada repetición se evaluaron 25 semillas; así, en esta prueba en total se evaluaron 150 semillas por tratamiento. Los resultados de las semillas con embriones viables, no viables, con vigor bajo y sin embrión fueron analizados mediante análisis de varianza, y la prueba de comparación múltiple de medias de Tukey $(\alpha=0.05)$.

\section{RESULTADOS}

En promedio no hubo diferencias significativas en el tamaño de las cápsulas recolectadas en 2012 y 2015 , ni en el número de semillas que contenían $(\mathrm{p}>0.05)$. La longitud promedio de las cápsulas fue cerca del doble de su diámetro y la biomasa de las semillas (45 $\mathrm{mg}$ ) por cápsula representó $38 \%$ de la biomasa total de las cápsulas. El coeficiente de variación confirmó la homogeneidad relativa entre las dimensiones, biomasa y número de semillas en las cápsulas de ambas recolectas (Cuadro 1). En contraste con la homogeneidad de las cápsulas, las semillas fueron parcialmente diferentes en sus dimensiones entre las recolectas. Las de 2015 fueron $17 \%$ más anchas que las de 2012 $(\mathrm{p} \leq 0.05)$, pero su longitud fue la misma ( $\mathrm{p}>$ 0.05 ); además, como resultado de su dimensión mayor, esas semillas pesaron $15 \%$ más que las de 2012 (Cuadro 1).

La diferencia en la proporción de semillas que germinó fue altamente significativa $(\mathrm{p} \leq$ 0.001) entre los años de recolecta. Para las muestras de 2012, la proporción de semillas que germinó y originó plántulas normales fue significativa $(\mathrm{p} \leq 0.05)$; pues representó solo $25 \%$ de las que germinaron en la segunda recolecta (Testigo Fig. 1A y Fig. 1B).

Los promotores de la germinación no modificaron estadísticamente $(\mathrm{p}>0.05)$ la proporción de semillas germinadas ni la cantidad de plántulas normales respecto al testigo de ninguna de las recolectas (Fig. 1 A y Fig. 1B).

CUADRO 1

Características de las cápsulas y semillas de dos recolectas de Hechtia perotensis, de la región de Frijol Colorado, municipio de Perote, Veracruz, México

TABLE 1

Characteristics of capsules and seeds of two collects of Hechtia perotensis, in the Frijol Colorado region, municipality of Perote, Veracruz, Mexico

\begin{tabular}{lccccc}
\multicolumn{1}{c}{ Cápsula } & Septiembre 2012 & Julio 2015 & Semillas & Septiembre 2012 & Julio 2015 \\
Diámetro $(\mathrm{mm})$ & $5.23 \mathrm{a}$ & $5.61 \mathrm{a}$ & Anchura & $1.26 \mathrm{~b}$ & $1.47 \mathrm{a}$ \\
& $(\mathrm{CV}=9.96 \%)$ & $(\mathrm{CV}=9.66 \%)$ & & $(\mathrm{CV} 14.19 \%)$ & $(\mathrm{CV}=16.64 \%)$ \\
Longitud $(\mathrm{mm})$ & $9.79 \mathrm{a}$ & $10.14 \mathrm{a}$ & Longitud & $3.57 \mathrm{a}$ & $3.58 \mathrm{a}$ \\
& $(\mathrm{CV}=7.08 \%)$ & $(\mathrm{CV}=8.31 \%)$ & & $(\mathrm{CV}=8.04 \%)$ & $(\mathrm{CV}=7.00 \%)$ \\
Biomasa $(\mathrm{mg})$ & $103.33 \mathrm{a}$ & $133.00 \mathrm{a}$ & Biomasa & $(\mathrm{CV}=460.06 \%)$ & $(\mathrm{CV}=31.05 \%)$ \\
& $(\mathrm{CV}=21.03 \%)$ & $(\mathrm{CV}=29.04 \%)$ & & & \\
Semillas por cápsula (Núm.) & $45.60 \mathrm{a}$ & $44.20 \mathrm{a}$ & & & \\
& $(\mathrm{CV}=23.34 \%)$ & $(\mathrm{CV}=18.48 \%)$ & & & \\
\hline
\end{tabular}

Letras iguales en los valores de cada variable de las dos recolectas indican que no hay diferencia entre ellos de acuerdo con la prueba de $t$ de Student; CV: coeficiente de variación. 


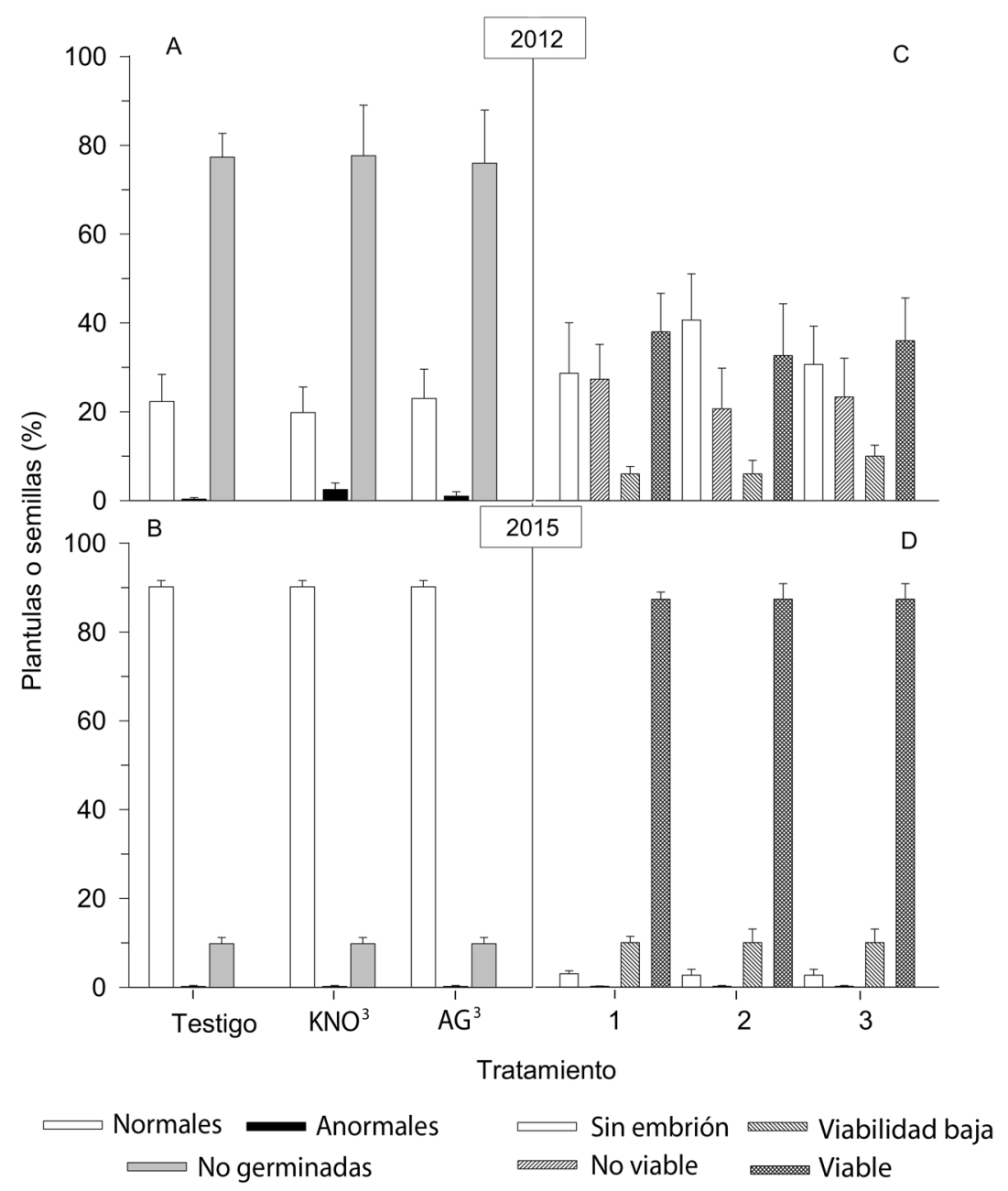

Fig. 1. Semillas que germinaron y generaron plántulas normales y anormales, semillas que no germinaron (A y B); semillas sin embrión, y con embrión con viabilidad baja, no viable o viable (C y D) en muestras de Hechtia perotensis, recolectadas en 2012 y 2015 en la región de Frijol Colorado, municipio de Perote, Veracruz, México. Cada columna representa el promedio de seis repeticiones, con 100 semillas cada una + error estándar.

Fig. 1. Seeds that germinated and produced normal and abnormal seedlings, non-germinated seeds (A and B); seeds without embryo, with embryo, low viability, non-viable and viable embryo (C and D) in samples of Hechtia perotensis collected in 2012 and 2015, in the Frijol Colorado region, municipality of Perote, Veracruz, Mexico. Each column represents average of six repetitions with 100 seeds each one + standard error.

La proporción de plántulas anormales no fue significativamente diferente $(\mathrm{p}>0.05)$ entre las recolectas (Fig. 1A y Fig. 1B). En la recolecta de 2012 la proporción de esas plántulas fluctuó entre 0.33 y $2.50 \%$ entre los tratamientos, y fue cerca de 20 veces menor a la proporción de plántulas normales, independientemente de la presencia o ausencia de los promotores de la germinación; pero, en promedio, el testigo presentó el porcentaje menor de ellas en comparación con los otros dos tratamientos (Fig. 1A). En la recolecta de 2015 no se observaron plántulas anormales en ninguno de los tratamientos (Fig. 1B).

La proporción de semillas que no germinaron fue significativamente diferente $(\mathrm{p} \leq 0.004)$ entre los años de recolecta, pero no existió diferencia significativa $(p>0.05)$ entre el testigo 
y los tratamientos con los promotores del crecimiento para cada recolecta (Fig. 1A y Fig. 1B). En la recolecta de 2012, dos tercios de la muestra no germinó en el tiempo de evaluación; y representó 4.5 veces la cantidad de semillas germinadas de la muestra de ese año (Fig. 1A). En contraste, en la muestra del año 2015, la proporción de semillas que no germinó fue poco menor de $10 \%$ (Fig. 1B).

Para conocer si la proporción de semillas no germinadas se debía a daño fisiológico del embrión o a embriones latentes, su viabilidad se cuantificó con la prueba de tetrazolio. En esta prueba fue indispensable que el corte en la testa de la semilla, previo al contacto con la solución de tetrazolio, se hiciera sin dañar el embrión; por lo que, para lograr lo anterior en semillas de $H$. perotensis con longitud menor a $4 \mathrm{~mm}$, fue indispensable cierta habilidad del evaluador. En este estudio, la prueba de tetrazolio permitió clasificar a las semillas de la recolecta del año 2012, en cuatro tipos según el color que adquirieron los embriones, que fueron: 1) semillas vigorosas, con embrión color rojo intenso en el tejido sano y, teóricamente, permeabilidad óptima de las membranas celulares; 2) semillas fisiológicamente sanas, en ellas los embriones se tiñeron con una coloración rosa y la solución de tetrazolio penetró lenta y uniformemente través las membranas celulares de sus tejidos; 3) semillas parcialmente dañadas o en proceso de deterioro, lesionadas o fisiológicamente envejecidas, con embriones teñidos de color rosa pálido y con zonas amplias no pigmentadas; y 4) semillas muertas, sin alguna tonalidad rojo-rosa, color blanco mate en el embrión completo. La clase 3 fue notablemente escasa en las semillas recolectadas en 2012 y no se presentó en las de 2015; esos embriones pigmentados parcialmente se contabilizaron como embriones muertos, de la clase 4. Así, los resultados de esta prueba permitió contabilizar, por diferencia de la muestra total, las semillas sin embrión o abortadas (Fig. 1C y Fig. 1D).

Además de conocer la capacidad germinativa de las semillas, basada en la presencia de embriones vivos, dañados o muertos (Fig. 1A y Fig. 1B) los resultados de la prueba con tetrazolio pueden relacionarse con los de la prueba de germinación estándar en laboratorio (Fig. 1C y Fig. 1D).

En la recolecta de 2012, la proporción de las semillas no fue significativamente diferente $(p>0.05)$ entre las clases (sin embrión, o con embrión no viable, con viabilidad baja o completamente viable). Este resultado fue independiente de la concentración de tetrazolio utilizada en la prueba. Por lo que, en promedio $36 \%$ de las semillas de la muestra tenían embrión viable, que al germinar podría haber expuesto las plántulas respectivas normales, además una proporción menor que $9 \%$ de la muestra, podrían haber germinado también en presencia de los promotores de la germinación. Asimismo, la muestra también incluyó una cantidad de semillas sin embrión, cercanamente similar a la de las semillas viables (Fig. 1C).

En contraste con las semillas recolectadas en 2012, en la recolecta de 2015 la proporción de las semillas fue significativamente diferente entre las clases ( $\mathrm{p} \leq 0.05)$, y este resultado fue independiente de la concentración de tetrazolio utilizada en la prueba. Así, esta última recolecta presentó en promedio $97.3 \%$ de semillas con embrión viable, pero $10 \%$ de ellas mostró viabilidad baja. Además, la muestra también incluyó proporciones significativamente menores de semillas sin embrión (2.67 \%), y aquellas con embrión no viable estuvieron prácticamente ausentes $(0.17 \%)$ (Fig. 1D). Ya que, la prueba de tetrazolio indicó que $36 \%$ de las semillas de la muestra era viable y la prueba estándar de germinación mostró únicamente $22 \%$ de germinación se deduce que la muestra recolectada en 2012 contenía cerca de $14 \%$ de semillas latentes.

Los factores ambientales, como la precipitación, la temperatura y la irradiancia, afectan la planta madre y la calidad de las semillas en desarrollo, lo que repercute en la capacidad germinativa de las semillas. Estos tres factores fluctuaron con tendencias diferentes en los meses previos a las recolectas de ambos años (Fig. 2A, Fig. 2B y Fig. 2C). En los 12 meses previos a la recolecta de 2012, la precipitación promedio mensual fluctuó entre 

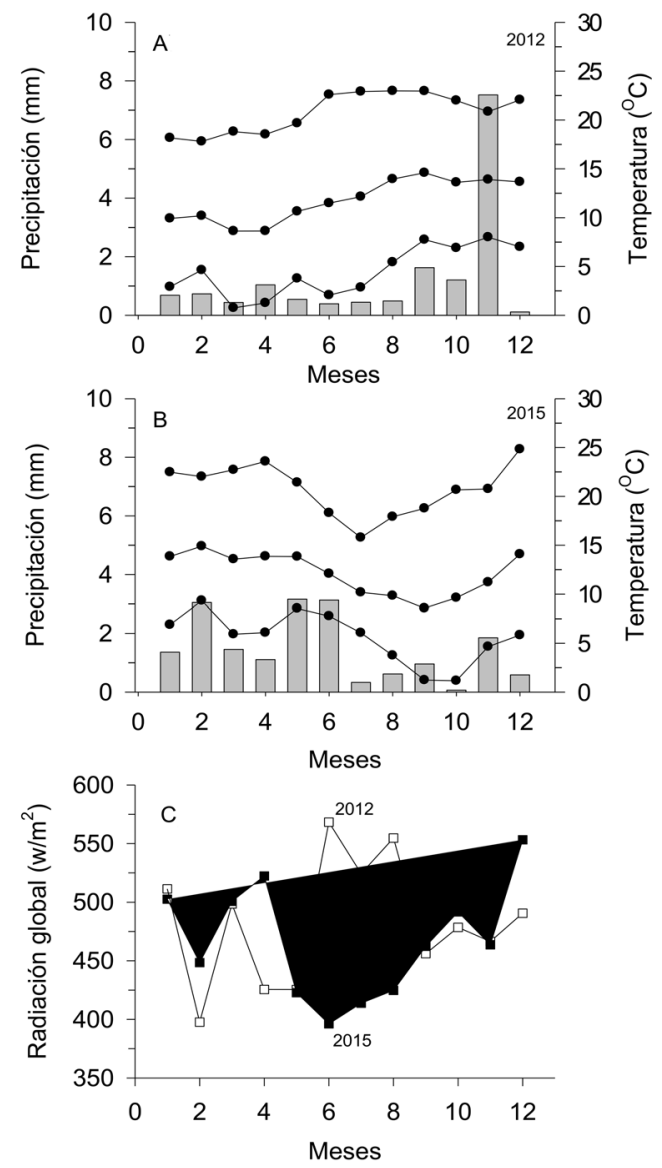

Fig. 2. Precipitación promedio $(\mathrm{mm})$, temperatura mínima, media y máxima mensual $\left({ }^{\circ} \mathrm{C}\right)(\mathrm{A}, \mathrm{B})$ y radiación global (C) en los 12 meses previos a recolección de semillas de Hechtia perotensis, en 2012 (A) y 2015 (B), en la región de Frijol Colorado, municipio de Perote, Veracruz, México. Fig. 2. Average rain $(\mathrm{mm})$, minimum, average and maximum temperature per month $\left({ }^{\circ} \mathrm{C}\right)(\mathrm{A}, \mathrm{B})$ and global sunlight $(\mathrm{C})$ in the previous 12 months to the Hechtia perotensis seed recollection, in 2012 (A) and 2015 (B), in the Frijol Colorado region, municipality of Perote, Veracruz, Mexico.

0.11 y $7.52 \mathrm{~mm}$; en contraste, en el caso de la recolecta de 2015, la fluctuación fue de 0.59 a 3.16 (Fig. 2A y Fig. 2B). En el caso de la precipitación, las fluctuaciones de la temperatura también fueron amplias y con patrones diferentes. Previo a la primera recolecta, las temperaturas promedio mensuales fluctuaron entre cerca de cero grados y $23{ }^{\circ} \mathrm{C}$, y los valores superiores a $20{ }^{\circ} \mathrm{C}$ se presentaron por seis meses (Fig. 2A). En contraste, antes de la segunda recolecta, las temperaturas promedio mensuales mostraron otras tendencias y medias mensuales máximas con fluctuación entre 15.8 y $25{ }^{\circ} \mathrm{C}$ (Fig. 2B). La radiación promedio global de los 12 meses previos a las recolectas (483 y $467 \mathrm{w} / \mathrm{m}^{2}$ en el 2012 y 2015) no fueron estadísticamente diferentes entre los años ( $\mathrm{p}=$ 0.438 ), pero los máximos y mínimos se presentaron en periodos diferentes. Las diferencias más sobresalientes se observaron en los meses 2, 4, 6 y 9; en los primeros la radiación global del 2012 fue $18 \%$ menor que la del 2015, y en el segundo periodo en 2015, la radiación global fue $25 \%$ menor que en el 2012 (Fig. 2C).

\section{DISCUSIÓN}

Las semillas de $H$. perotensis evaluadas en este estudio fueron similares en color, longitud y anchura independientemente de la fechas de recolecta, pero provenientes de la misma localidad Perote, Veracruz, México. Además, esos atributos seminales coincidieron con los descritos por otros investigadores en las semillas de otra localidad (Aljojuca, Puebla, México; km 15 carretera federal San Salvador El SecoEsperanza, 1905'33" N - 97³2'17” W, 2500 msnm) y fechas (Espejo-Serna et al., 2007). Los resultados indicaron que esos atributos fueron notablemente estables entre las semillas y poblaciones de $H$. perotensis.

En contraste, la capacidad germinativa fue significativamente diferente entre los años de recolecta. En 2012 la prueba de germinación estándar indicó que $77 \%$ de la muestra no tenía capacidad para germinar, durante los primeros $15 \mathrm{~d}$, en las condiciones de temperatura, luz y humedad y oxígeno en laboratorio, que se consideraron óptimas o estándar para este proceso (International Seed Testing Association, 2010). Pero en la muestra de 2015, las semillas inviables estuvieron prácticamente ausentes. Las diferencias entre los años de recolecta de este estudio confirmaron indirectamente el riesgo de permanencia de la población endémica de $H$. perotensis, en la localidad de recolecta, dependiente de la calidad de las semillas 
y su capacidad para germinar y generar plantas normales en algunos ciclos de producción de semilla. Aunque no se tiene evidencia, podría sugerirse que algo similar sucede en las demás poblaciones hasta ahora conocidas del centrooeste de Veracruz y su porción limítrofe con Puebla, México (Espejo-Serna et al. 2007).

Las características germinativas de las especies silvestres y endémicas, como $H$. perotensis, son relevantes por diversas causas. La germinación de las semillas está entre los eventos más tempranos y drásticos del desarrollo que suceden en la vida de las plantas, pues con él pasan de ser organismos quiescentes a activos, de estar protegidos con una testa a exponerse a todos los elementos del ambiente y de ser heterótrofos a autótrofos (Donohue, Rubio de Casas, Burghardt, Kovach, \& Willis, 2010). Este estudio reveló que las semillas de la muestra de $H$. perotensis recolectadas en 2012, no fueron vigorosas, y las recolectadas tres años después expresaron vigor máximo; pero esta prueba no aporta información sobre aspectos como la adaptación o la distribución geográfica de la especie, por lo que para continuar esta investigación se espera complementar la información con otros análisis.

La prueba estándar de germinación en laboratorio tampoco ayuda a discriminar entre las semillas que no germinaron por ser latentes, abortadas, o tener embrión dañado. En contraste, la prueba con tetrazolio, independientemente de la concentración usada, permitió determinar que las semillas de la muestra analizadas tenían potencial germinativo contrastante e indicaron que en promedio $64 \%$ de las semillas de la muestra de 2012 no tenían posibilidad de germinar o solo una proporción del $7 \%$ podría haberlo hecho si las condiciones de germinación hubieran sido totalmente favorables para activar los embriones con viabilidad baja de esas semillas.

Los resultados, también permitieron calcular la muestra recolectada en 2012 contenía semillas latentes puesto que el porcentaje de semillas viables obtenidas en la prueba de tetrazolio es mayor al porcentaje de semillas germinadas obtenidas en la prueba estándar de germinación. En contraste, las semillas de la recolecta de 2015 no presentaron latencia, la proporción de semillas que fue clasificada como vigorosa, con la prueba de tretrazolio, germinó en la prueba estándar.

La proporción de semillas germinadas, latentes y no latentes en las poblaciones, como la de $H$. perotensis muestreada para el presente estudio, permite conocer indirectamente las características del banco de semillas. En la muestra de 2012, en promedio, de las 46 semillas por cápsula solo 17 fueron viables, ocho podrían haberse encontrado en estado latente y el resto eran semillas abortadas o muertas. En contraste, la de 2015 en promedio una cápsula tendría casi exclusivamente semillas viables y únicamente una semilla no viable o abortada.

La latencia es una propiedad de la semilla que previene su germinación cuando está en condiciones favorables para ella. Este fenómeno es influenciado por la información genética de la especie, las condiciones ambientales, el balance de reguladores del crecimiento, como ácido abscísico (ABA) y giberelinas, y la interacción de estos dos con el etileno, y algunas auxinas o brasinoesteroides (Bewley, 1997; Finkelstein, Gampala \& Rock, 2002; FinchSavage \& Leubner-Metzger, 2006). La latencia puede estar relacionada con el embrión o con las estructuras que lo rodean, como la testa, lo que permite distinguir varias clases de latencia (Finch-Savage \& Leubner-Metzger, 2006). En el caso de $H$. perotensis, independientemente del tipo de latencia, la proporción de este tipo de semillas cambió significativamente entre las recolectas y notablemente no existió en la muestra recolectada en 2015. Este resultado podría deberse al efecto de factores diversos y variados, tanto intrínsecos de la planta y sus estructuras, como de los ambientales durante su desarrollo y maduración, y a la interacción de ellos, como se ha observado en otras especies silvestres (Peña-Valdivia et al., 2006). La calidad menor de las semillas recolectadas en 2012 respecto a las de 2015, parece estar relacionada con la escasa precipitación (menos a $1.03 \mathrm{~mm}$ mensuales) durante nueve meses previos a las recolectas; en contraste, la precipitación 
antes de la recolección de 2015 fue más abundante, frecuente y regular.

También la temperatura mínima, máxima y media durante el año que antecedió a las recolectas, mostró patrones distintos entre los años y mínimos y máximos parcialmente contrastantes. En el caso de las temperaturas mínimas mensuales previas a la recolección de 2012, durante ocho meses fueron cercanas a $0{ }^{\circ} \mathrm{C}$ y menores a $5{ }^{\circ} \mathrm{C}$. En contraste, 10 meses previos a la recolección de 2015 fueron más cálidos y su temperatura mínima fluctuó entre 5 y $10^{\circ} \mathrm{C}$. Además, la temperatura ambiente máxima en el ciclo previo a la recolección de 2012, también mostró cinco meses relativamente fríos, con temperaturas máximas menores de $20{ }^{\circ} \mathrm{C}$. En contraste, la mayoría de los meses previos a la recolección de 2015, mostraron temperaturas máximas que fluctuaron alrededor de $\operatorname{los} 22^{\circ} \mathrm{C}$, y algunos mostraron hasta 24 y $25^{\circ} \mathrm{C}$. En consecuencia, los patrones de temperatura media mensual entre los dos periodos analizados mostraron tendencias diferentes.

Aunque actualmente no se tiene información de la fenología de $H$. perotensis, la información anterior es evidencia del efecto de la precipitación y la temperatura ambiente durante la fructificación y probablemente durante la floración de las plantas de la población estudiada.

La depredación de semillas es con frecuencia una causa importante de mortalidad (Wenny, 2000), y si las condiciones son favorables para el crecimiento de las plántulas entonces la germinación rápida podría ser ventajosa (Xiao, Harris, \& Zhang, 2007). La viabilidad en el banco de semillas es necesaria para que la latencia sea adaptable, o las semillas pueden morir antes de germinar, así que el valor adaptativo de la latencia depende del riesgo relativo de mortalidad de las semillas en el suelo, frente a la mortalidad de las plántulas. La longevidad intrínseca de la semilla en el suelo también se convierte en un problema. Si las semillas tienen pocas reservas o están metabólicamente muy activas para soportar períodos largos antes de la germinación, la inactividad prolongada conduciría a la muerte de la semilla (Donohue et al., 2010). En este contexto, la depredación de semillas, el tiempo de germinación, las características del banco de semillas ni la longevidad de estas no se ha documentado en $H$. perotensis; lo que confirma la importancia de continuar evaluando las poblaciones de esta especie endémica.

El desarrollo de las plantas y sus estructuras, incluidas las semillas, depende de la radiación, ya que la radiación baja puede limitar drásticamente la disponibilidad de $\mathrm{C}$ y con esto se restringen los suministros de energía para procesos metabólicos esenciales, como gradientes celulares y síntesis de proteínas, y las plantas deben alcanzar un equilibrio entre la asignación de $\mathrm{C}$ para el crecimiento y la acumulación de reservas (Pons \& Poorter, 2014). Lo anterior y la información actual permiten sugerir que la radiación y su interacción con otros factores ambientales, también pudo haber afectado la calidad fisiológica de las semillas de H. perotensis evaluadas.

En general, los promotores germinativos $\mathrm{KNO}^{3}$ y $\mathrm{AG}^{3}$ no modificaron el porcentaje de germinación; pero, en la primera recolecta sí hubo un incremento pequeño de semillas germinadas que originaron plántulas anormales. Estos resultados permitieron confirmar los resultados de la proporción de las semillas latentes en esa muestra.

Estos resultados coincidieron con los de Amador-Alférez, Díaz-González, Loza-Cornejo, y Bivián-Castro (2013) con semillas de F. histrix y F. latispinus (Cactaceae); en los que no obtuvieron incremento significativos de los porcentajes de germinación de semillas con la aplicación de $500 \mathrm{mg} / \mathrm{L}$ de $\mathrm{AG}^{3}$ en comparación con el testigo. El $\mathrm{AG}^{3}$ tampoco tuvo efecto en la germinación de Opuntia rastrera, $O$. microdasys y O. macrocentra (Mandujano, Golubov, \& Rojas-Aréchiga, 2007). Los resultados de la recolecta de 2012 difirieron de los de Magnitskiy y Ligarreto (2007) con semillas de agraz (Vaccinium meridionale Swartz), las que sí incrementaron (32 \%) su germinación después de $35 \mathrm{~d}$, con la imbibición de las semillas en soluciones acuosas de $\mathrm{KNO}^{3}$ y $\mathrm{AG}^{3}$. Este resultado puede ser indicativo 
de que la falta de efecto de $\mathrm{AG}^{3}$ y $\mathrm{KNO}^{3}$ en las semillas de $H$. perotensis podría cambiar con otras concentraciones diferentes a las utilizadas en este estudio. Los efectos positivos en el incremento de la germinación, emergencia y en la sincronía de estos eventos se han logrado con la imbibición de las semillas con $200 \mathrm{mg} / \mathrm{L}$ (Navarrete, 1996) a $600 \mathrm{mg} / \mathrm{L}$ de $\mathrm{AG}^{3}$ (Furatani \& Nagao, 1987). Lima, Lima, Venezuela, y Macias (1985) mencionaron que al aplicar AG (1.0 mM) se inhibió la latencia de las semillas y se eliminó el efecto de las sustancias lipoproteícas que retardaron o inhibieron su germinación. Davière y Achard (2013) describieron los procesos de señalización de las giberelinas en las plantas. Estos autores señalaron que las proteínas denominadas DELLA fueron las represoras centrales de los procesos dependientes de AG, como la germinación; el genoma de Arabidopsis codificó cinco DELLAs que desarrollaron distintas funciones en las respuestas a AG, de ellas, específicamente las conocidas como RGL2 inhibieron la germinación de las semillas. Además, el descubrimiento de un receptor GA (GID1) permitió reconocer que la ausencia de DELLA conduce a la inhabilidad de DELLAs para interaccionar con GID1 aun en la presencia de AG. Por lo que, no todas las especies y variantes responderán a los compuestos denominados genéricamente promotores de la germinación.

Otro aspecto fue el referente a la prueba de viabilidad, la cual nos indicó que las concentraciones de tetrazolio evaluadas no generaron diferencia significativa $(\mathrm{p}>0.05)$ en la tinción de los embriones y todas permitieron cuantificar proporciones significativamente similares de cada clase de semillas, con embrión viable, sin él o con vigor bajo. Podría asegurarse que para $H$. perotensis el uso de cualquier concentración de este compuesto, entre 0.2 y $1.0 \%$ es adecuada para conocer la viabilidad de los embriones. Estos resultados coincidieron con los de Craviotto et al. (2011), ya que con solución de tetrazolio al 0.1 y $0.5 \%$, a $30{ }^{\circ} \mathrm{C}$ por $3 \mathrm{~h}$, obtuvieron en semillas de soya resultados adecuados para discernir la coloración con matices diferentes, que fueron interpretados como indicativos de su estado fisiológico. Aunque no hubo diferencias significativas ( $p>$ 0.05 ) entre las concentraciones en las muestras de cada recolecta en el presente estudio, debe indicarse que con tetrazolio al $0.2 \%$ hubo cierta dificultad para diferenciar entre los embriones viables y los no viables de la recolecta de 2015 .

Victoria, Bonilla, y Sánchez (2006) utilizaron solución de tetrazolio al $0.5 \%$, por tiempos y temperaturas diferentes, en semillas de Calendula officinalis y lograron la tinción solo de los cotiledones. Además, en semillas de Anethum graveolens lograron la tinción adecuada del embrión con tetrazolio al $1 \%$ y la de la semilla completa con $0.5 \%$. Los resultados coincidieron con los obtenidos en las semillas de $H$. perotensis, en las que la concentración de tetrazolio 0.5 a $1 \%$ permitió discernir entre embriones viables y no viables. Esto indica que para las semillas de cada género y especie es necesario un estudio detallado que incluya tiempos, temperaturas y concentraciones variables del colorante, para establecer los patrones de coloración de los tejidos.

La proporción de semillas viables y no viables y latentes de $H$. perotensis varía su calidad fisiológica en dependencia del año de producción; esa calidad es dependiente del clima, como la precipitación y las temperaturas mínimas, máximas y medias, además también puede evidenciarse el efecto directo de la radiación global. Las semillas de la especie en estudio con potencial germinativo bajo no se modifican por promotores de la germinación. El protocolo de la prueba de tetrazolio es adecuado para evaluar la calidad de los embriones en semillas de esa especie de Hechtia, pues los resultados coinciden con los de la germinación.

\section{AGRADECIMIENTOS}

Los autores agradecemos a Gerardo Benjamín Torres Cantú, estudiante de doctorado del Posgrado en Botánica del Colegio de Postgraduados, por identificar las especies del sitio de recolecta. 


\section{RESUMEN}

Poblaciones endémicas de Hechtia perotensis se han descrito en Puebla y Veracruz, México. La recolección de semillas de buena calidad permite su uso en conservación, investigación y restauración ecológica. Algunos compuestos para evaluar la calidad de las semillas silvestres y endémicas, como nitrato de potasio $\left(\mathrm{KNO}^{3}\right)$ y ácido giberílico $\left(\mathrm{AG}^{3}\right)$, se utilizan para incrementar la germinación de las semillas y disminuir la latencia. La prueba de cloruro de trifeniltetrazolio (tetrazolio) se correlaciona con la viabilidad de las semillas porque se basa en la actividad de las deshidrogenasas de tejidos vivos que catalizan la respiración mitocondrial. El objetivo de este estudio fue conocer el tamaño y el peso de las cápsulas y las semillas y la germinación y viabilidad de las semillas de $H$. perotensis recolectadas en Veracruz en 2012 y 2015. Las hipótesis fueron (1) que la germinación y la viabilidad de las semillas son independientes del año de recolecta, (2) que hay una concentración adecuada de tetrazolio para identificar la viabilidad de las semillas y (3) que el pretratamiento con $\mathrm{KNO}^{3}$ o $\mathrm{AG}^{3}$ incrementa la germinación de las semillas. La germinación se evaluó con un diseño completamente al azar con tres tratamientos (testigo y promotores de la germinación $\mathrm{KNO}^{3} 0.2 \%$ y $\mathrm{AG}^{3} 500 \mathrm{mg} / \mathrm{L}$ ), cuatro tratamientos para la prueba de viabilidad (testigo, $0.2,0.5$ y 1.0 $\%$ de tetrazolio) y seis repeticiones de cada tratamiento. La germinación se evaluó en 100 semillas y la viabilidad en 25. Los resultados entre y dentro de años se analizaron con ANDEVA y prueba de comparación múltiple de medias de Tukey. La proporción de semillas no germinadas se cuantificó junto con el número de plántulas normales y anormales, semillas con embrión viable o sin él, y con viabilidad baja o sin viabilidad. En promedio la muestra recolectada en 2012 tuvo $36 \%$ de semillas con embrión viable, 7 con viabilidad baja, $24 \%$ no viables y $33 \%$ sin embrión. Este resultado fue significativamente diferente al de 2015, que presentó $87 \%$ de embriones viables, $10 \%$ con viabilidad baja, $0 \%$ no viables y $3 \%$ sin embrión. La germinación también fue significativamente diferente entre los años (22 y $92 \%$ ). Los tratamientos pregerminativos no modificaron la germinación. La germinación y la viabilidad de las semillas de $H$. perotensis varían significativamente entre los años de recolecta.

Palabras clave: Bromeliaceae, Hechtia perotensis, latencia, semilla, tetrazolio, vigor de semilla.

\section{REFERENCIAS}

Amador-Alférez, K. A., Díaz-González, J., Loza-Cornejo, S., \& Bivián-Castro, E. Y. (2013). Efecto de diferentes reguladores de crecimiento vegetal sobre la germinación de semillas y desarrollo de plántulas de dos especies de Ferocactus (Cactaceae). Polibotánica, 35, 109-131.
Baskin, C. C., \& Baskin, J. M. (2008). Advances in understanding seed dormancy at the whole-seed level: An ecological, biogeographical and phylogenetic perspective. Acta Botanica Yunnanica, 30, 279-294.

Bewley, J. D. (1997). Seed germination and dormancy. The Plant Cell, 9, 1055-1066.

Craviotto, R. M., Arango, P. M. R., \& Gallo, C. (2011). Novedades de la Prueba de Viabilidad por Tetrazolio en Soja. Santa Fe, Argentina: Instituto Nacional de Tecnología Agropecuaria. Laboratorio de semillas EEA Oliveros.

Davière, J. M., \& Achard, P. A. (2013). Gibberellin signaling in plants. Development, 140, 1147-1151.

Deno, N. C. (1994). The critical role of gibberellins in germination and survival of certain cacti. Cactus and Succulent Journal, 66, 28-30.

Donohue, K., Rubio de Casas, R., Burghardt, L., Kovach, K., \& Willis, C. G. (2010). Germination, postgermination adaptation, and species ecological ranges. Annual Review of Ecology, Evolution, and Systematic, 41, 293-319.

El-Maarouf-Bouteau, H., \& Bailly, C. (2008). Oxidative signaling in seed germination and dormancy. Plant Signaling \& Behavior, 3, 175-182.

Escobedo, S., J. (2012). Mecanismos de dispersión de semillas en las Bromelias. Desde el herbario CICY 4: 22-23. http://www.cicy.mx/sitios/desde_herbario/

Espejo-Serna, A., López-Ferrari, A. R., Ramírez-Morillo, I., \& Martínez-Correa, N. (2007). Dos nuevas especies de Hechtia (Bromeliaceae) de México. Acta Botánica Mexicana, 78, 97-109.

Finch-Savage, W. E., \& Leubner-Metzger, G. (2006). Seed dormancy and the control of germination. New Phytologist, 171, 501-23.

Finkelstein, R. R., Gampala, S. S., \& Rock, C. D. (2002). Abscisic acid signaling in seeds and seedlings. The Plant Cell Supplement, 14, 15-45.

Furatani, S. C., \& Nagao, M. A. (1987). Influence of temperature, $\mathrm{KNO}^{3}, \mathrm{AG}^{3}$ and seed drying on emergence of papaya seedlings. Scientia Horticulturae, $32,67-72$.

García, E. (1988). Modificaciones al sistema de clasificación climática de Köppen. México: Universidad Nacional Autónoma de México.

García, F. A., Montes, H. S., Rangel, L. J. A., García, M. E., \& Mendoza, E. M. (2010). Respuesta fisiológica de la semilla chile piquín [Capsicum annuum var. glabriusculum (Dunal) Heiser \& Pickersgill] al ácido giberélico e hidrotermia. Revista Mexicana de Ciencias Agrícolas, 1, 203-216. 
Givnish, T. J., Millam, K. C., Berry, P. E., \& Sytsma, K. J. (2007). Phylogeny, adaptive radiation, and historical biogeography of Bromeliaceae inferred from ndhf sequence data. Aliso, 23, 3-26.

Glenner, G. G. (1990). Formazans and tetrazolium salts. En R. D. Lillie (Ed.), Biological Stains (pp. 225-235). St. MO: Sigma Chemical Company.

International Seed Testing Association (2010). International Rules for Seed Testing. Basserdorf, CH Switzerland.

Lima, D. S., Lima, D. I., Venezuela, G. R., \& Macias, P. (1985). Estudio de la viabilidad de las semillas de Carica papaya L. (Variedad de Maradol Roja). Centro Agrícola, 12, 119-130.

Llorente-Bousquets, J., \& Ocegueda, S. (2008). Estado del conocimiento de la biota, en Capital natural de México (vol. I, pp. 283-322). Conocimiento actual de la biodiversidad. México: Conabio.

Magnitskiy, S. V. \& Ligarreto, G. A. (2007). Efecto del nitrato de potasio, del ácido giberélico y del ácido indolacético sobre la germinación de semillas de agraz (Vaccinium meridionale Swartz). Revista Colombiana de Ciencias Hortícolas, 1, 137-141.

Mandujano, M. C., Golubov, J., \& Rojas-Aréchiga, M. (2007). Efecto del ácido giberélico en la germinación de tres especies del género Opuntia (Cactaceae) del Desierto Chihuahuense. Cactáceas y Suculentas Mexicanas, 52, 46-52.

Montes, R. S. (2013). Germinación de especies de la familia Bromeliaceas: aspectos ecológicos y anatómicos (Tesis de Doctorado). Universidad Autónoma de México, México.

Navarrete, R. J. A. (1996). Producción del Papayo (Carica papaya L.) Variedad Maradol en Yucatán. Instituto Tecnológico Agropecuario No. 2. Mérida, Yucatán. México: Centro de Investigación y Graduados Agropecuarios. Dirección General de Educación Tecnológica Agropecuaria.

Peña-Valdivia, C. B., Sánchez-Urdaneta, A. B., Aguirre, R. J. R., Trejo, C., Cárdenas, E., \& Villegas, M. A (2006). Temperature and mechanical scarification on seed germination of "maguey" (Agave salmiana Otto ex Salm-Dyck). Seed Science and Technology, $34,47-56$.

Pereira, A. R., Andrade, A. C. S., Pereira, T. S., Forzza, R. C., \& Rodríguez, A. S. (2010). Morphological aspects of seed, germination and storage of Pitcairnia albiflos (Bromeliaceae). Seed Science and Technology, 38, 79-87.

Pons, T. L., \& Poorte, H. (2014). The effect of irradiance on the carbon balance and tissue characteristics of five herbaceous species differing in shade-tolerance. Frontiers in Plant Science, 5, 1-14.

Ramírez, M. I., Jiménez, C. F., Fernández-Concha, G. C., \& Pinzón, J. P. (2014). Three new species and growth patterns in Hechtia (Bromeliaceae: Hechtioideae). Phytotaxa, 178, 113-127.

Rzedowski, J. (1991). El endemismo de la flora fanerogámica mexicana: una apreciación analítica preliminar. Acta Botánica Mexicana, 15, 47-64.

Reyes, S. J. (2015). Conservación y restauración de cactáceas y otras plantas suculentas mexicanas. México: Comisión Nacional Forestal.

Saldívar-Iglesias, P., Laguna-Cerda, A., Gutiérrez-Rodríguez, F., \& Domínguez-Galindo, M. (2010). Ácido giberélico en la germinación de semillas de Jaltomata procumbens (Cav.) J. L. Gentry. Agronomía Mesoamericana, 21, 327-331.

Sarukhán, J., Halffter, G., \& Koleff, P. (2009). Capital natural de México. Síntesis: conocimiento actual, evaluación y perspectivas de sustentabilidad. México: Comisión Nacional para el Conocimiento y Uso de la Biodiversidad.

Siadat, S. A., Moosavi, S. A., Zadeh, M. S., Fotouhi, F., \& Zirezadeh, M. (2011). Effects of halo and phytohormone seed priming on germination and seedling growth of maize under different duration of accelerated ageing treatment. African Journal of Agricultural Research, 6, 6453-6462.

Sosa, L. D., Chávez, S. J. L., Mondragón, Ch. D., Estrada, G. J. A., \& Ramírez, V. P. (2012). Viabilidad y germinación de semillas de seis especies de Tillandsia (Bromeliaceae) de Oaxaca, México. Fitotecnia Mexicana, 35, 37-42.

Tigabu, M., \& Oden, P. C. (2001). Effect of scarification, gibberellic acid and temperature on seed germination of two multipurpose Albizia species from Ethiopia". Seed Science and Technology, 29, 11-20.

Toledo, A. T., \& Wolf, J. H. D. (2008). Germination and establishment of Tillandsia eizii (Bromeliaceae) in the canopy of an oak forest in Chiapas, Mexico. Biotropica, 40, 246-250.

Victoria, J., Bonilla, C., \& Sánchez, M. (2006). Viabilidad en tetrazolio de semillas de caléndula y eneldo. Acta Agronómica, 55, 14-18.

Wenny, D. G. (2000). Seed dispersal, seed predation, and seedling recruitment of a neotropical montane tree. Ecological Monograph, 70, 331-351.

Xiao, Z. S., Harris, M. K., \& Zhang, Z. B. (2007). Acorn defenses to herbivory from insects: implications for the joint evolution of resistance, tolerance and escape. Forest Ecology and Management, 238, 302-308.

Zheng, C., Jiang, D., Liub, F., Dai, T., Liu, W., Jing, Q., \& Cao, W. (2009). Exogenous nitricoxide improves seed germination in wheat against mitochondrial oxidative damage induced by high salinity. Environmental and Experimental Botany, 67, 222-227. 
